\title{
Endoscopic management of pediatric brain tumors
}

\author{
Mark M. Souweidane, M.D. \\ Departments of Neurological Surgery and Pediatrics, Weill Medical College of Cornell University \\ and Memorial Sloan-Kettering Cancer Center, New York, New York
}

\begin{abstract}
Object. Primary endoscopic procedures for children with intraventricular brain tumors include endoscopic tumor biopsy and endoscopic tumor removal. The simultaneous treatment of hydrocephalus with endoscopic third ventriculostomy (ETV) or endoscopic septostomy increases the appeal of a minimally invasive endoscopic approach.

Methods. Eighty-five patients who underwent endoscopic management of an intraventricular brain tumor were identified from a prospective database. Of these patients, 26 were younger than 21 years of age at the time of diagnosis. The surgical technique, its success rate, and patient outcome were assessed. Illustrative cases are used in this study to detail the procedure of endoscopic tumor biopsy and resection.

Endoscopic tumor procedures were successful in $96 \%$ of cases ( 23 of 24 endoscopic tumor biopsy samples and both endoscopic tumor removals). Fourteen simultaneous procedures were performed to treat hydrocephalus successfully. There was no recognized morbidity from the surgical procedures.

Conclusions. Endoscopic surgery in children with intraventricular brain tumors is an effective and safe method for sampling of the lesion and, in select cases, its resection. This minimally invasive technique should be considered in situations in which the patient might thereby avoid a more conventional procedure, given the high rate of success and low morbidity associated with endoscopic management.
\end{abstract}

KEY WORDS • endoscopy • brain tumor • colloid cyst • hydrocephalus

With the advent of contemporary neuroendoscopy, which was heralded by advances in lens technology and light intensity, the indications for endoscopic neurosurgery have grown. The utility of endoscopic surgery is well established for the treatment of noncommunicating hydrocephalus, compartmentalized hydrocephalus, and arachnoid cysts. ${ }^{3,8}$, 13,16,21,23 Endoscopic neurosurgery for intraventricular brain tumors is a natural evolution of the technology, given that these lesions are positioned within a CSF interface and are amenable to detailed endoscopic inspection. Further contributing to the appeal of primary endoscopic management of intraventricular lesions is the fact that a number of tumors in children will not ultimately require aggressive surgical removal and that conventional craniotomy procedures can be demanding, with an inherently higher rate of surgical morbidity. Last, because of the concomitant finding of CSF obstruction, the majority of patients can potentially benefit from simultaneous endoscopic procedures aimed at symptomatic relief of hydrocephalus, such as ETV and endoscopic septostomy. Thus, primary endoscopic tumor biopsy and endoscopic tumor resection are becoming better characterized as procedures that can positively contribute toward the management of intraventricular brain tumors in children.

Abbreviations used in this paper: $\mathrm{CSF}=$ cerebrospinal fluid; $\mathrm{ETV}=$ endoscopic third ventriculostomy; $\mathrm{MR}=$ magnetic resonance.

\section{CLINICAL MATERIAL AND METHODS}

\section{Patient Population}

Between December 1995 and March 2005, I treated 85 patients with intraventricular brain tumors were treated by using a primary endoscopic technique. Patients in whom an endoscopically assisted surgery was performed are not included in this review. From this patient cohort, those individuals 21 years of age or younger were selected for analysis. The surgical technique, the success rate, and patient outcome were assessed. The Institutional Review Board/ Privacy Board at Weill Medical College of Cornell University and Memorial Sloan-Kettering Cancer Center granted approval for the review of medical records and completion of this study.

\section{Surgical Procedure}

Endoscopic tumor biopsy and resection have previously been reported. ${ }^{2,22}$ All procedures were performed after induction of general anesthesia. No seizure prophylaxis or steroid therapy was used. All patients received intravenous antibiotic prophylaxis prior to skin incision. To begin the procedure, the patient was positioned supine with the head elevated to approximately $30^{\circ}$ above the horizontal in an effort to minimize CSF egress from the endoscope. For procedures in which frameless stereotaxy was used, the patient's skull was secured with pin fixation. A burr hole was used 
for access, with the entry site dependent on the location of the tumor and the need to perform a concurrent procedure (that is, third ventriculostomy or septostomy). $\mathrm{A}^{\circ}$ or $30^{\circ}$ rigid lens endoscope (MINOP; B. Braun Aesculap, Tuttlingen, Germany) was used for the initial ventricular cannulation. The endoscopic sheath has an outside diameter of $6 \mathrm{~mm}$ and a 2-mm working channel. When stereotaxy was needed for trajectory planning, the sheath was registered using an optical guided stereotactic system (Stealth; Medtronic, Inc., Minneapolis, MN; Fig. 1).

Surgical planning is critical for successful endoscopic tumor procedures. The most critical aspect is selecting an entry site that offers the most direct intraventricular, linear route to the target (Fig. 2). This principle avoids undue torque on the cortical and intraventricular surfaces. A relatively anterior entry site with reference to the coronal suture is most important for lesions situated in the posterior third ventricle or pineal region. With respect to laterality, most entries should be placed on the nondominant side. Exceptions to this recommendation would include the following: 1) hypothalamic lesions, which are best targeted using a contralateral approach; and 2) in situations in which there is significant ventricular asymmetry, in which case the preference is to enter the side with the greater ventricular size. Once the burr hole is made, it is helpful to create a conical shape on its outside by using a high-speed drill. This simple maneuver allows greater variability in the trajectory and maneuverability of the scope.

Following entry into the ventricular compartment, normal anatomical landmarks, including the choroid plexus, venous tributaries, and the septum pellucidum are used to establish appropriate orientation. Inspection of the ependymal surface of the ventricle is performed to assess any metastatic components of the disease. Once the lesion is identified, cupped biopsy forceps are used for tissue sampling. The sites for this procedure are chosen based on an avascular pattern and the appearance of the tumor surface. Given the limited sample size, coagulation of the tumor surface prior to tissue sampling is intentionally avoided to guard against any thermal artifact. If further removal of the tumor is to be attempted, the resection is then conducted using appropriate instruments. Following a generous coagulation

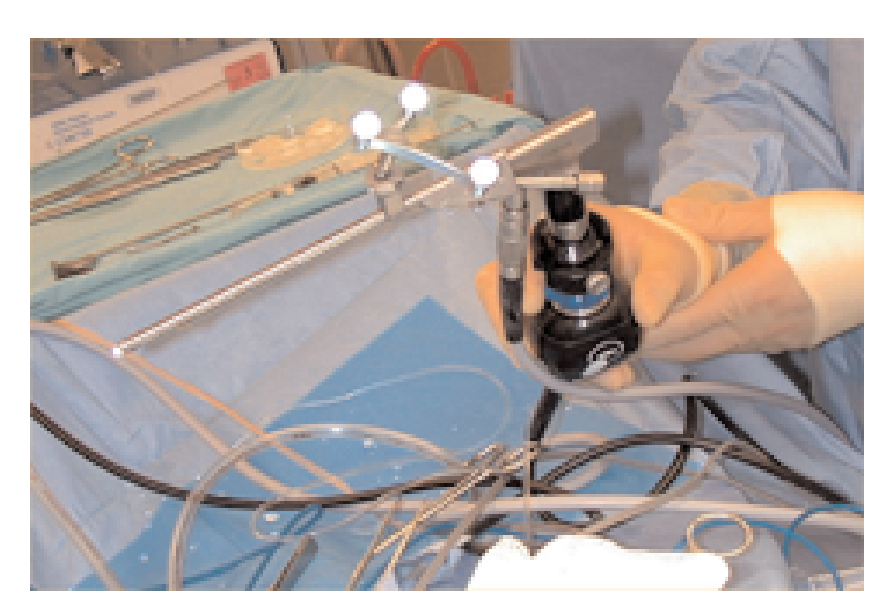

Fig. 1. Photograph of a rod lens endoscope coupled with a tracking device for optically guided stereotaxy.

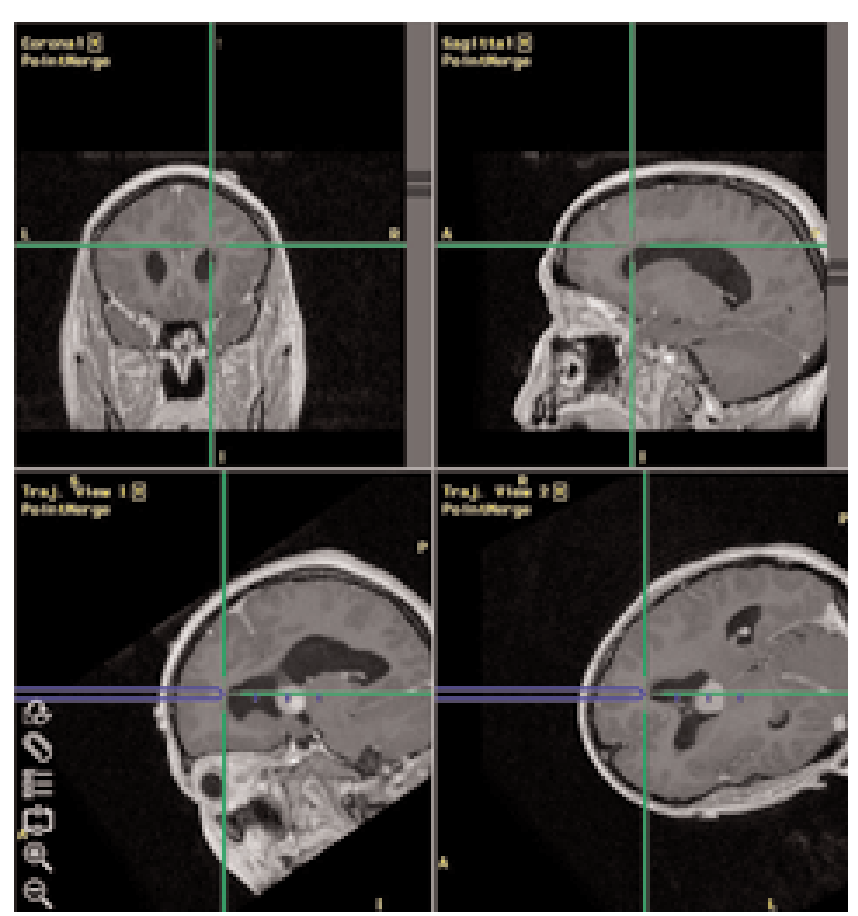

Fig. 2. A typical screen capture obtained during stereotactically guided endoscopic ventricular cannulation. The top row of images indicates the orthogonal position of the endoscope, whereas the bottom row depicts its trajectory. In this particular case, the optimal trajectory was chosen to approximate the roof of the third ventricle, which is the site of attachment of colloid cysts.

of the tumor surface, a No. 6 French endotracheal suction catheter is used to remove friable tissue or cystic contents with a pulsed technique. Aspiration is only applied once the catheter tip is firmly and completely embedded within the tumor tissue. The degree of suction is regulated to act preferentially on friable tumor tissue rather than the more solid parenchymal interface. This maneuver is alternated with blunt dissection to separate the tumor surface from adjacent ependymal tissue. Any recognized tumor remnants are then generously coagulated with bipolar diathermy (Video 1).

\footnotetext{
Click here to view the video clip: Endoscopic resection of a posterior tinira ventricle epenaymoma. A right-sided approach is used for initial entry into the frontal horn of the lateral ventricle. Immediately identified are the normal anatomical structures of the intraventricular compartment, including the foramen of Monro, the choroid plexus, the fornix, and the septal and thalamostriate veins. The endoscope is then navigated through a widened foramen of Monro, with care being taken to avoid the choroid plexus. The paired mammillary bodies and the blue membrane of the floor of the third ventricle can be seen beyond the foramen. The $30^{\circ}$-angled endoscope is then repositioned so that the roof of the third ventricle will be visible. As this adjustment is made, the choroid plexus emanating from the contralateral foramen of Monro is visible. As the endoscope is moved into the posterior third ventricle, the massa intermedia is seen on the left and the tumor comes into view below this commissure. The lesion, which is clearly distinguished from the surrounding ependyma, is situated at the level of the cerebral aqueduct. Bipolar diathermy is used generously to coagulate the surface of the mass. An aspiration catheter is then used to empty any cystic or semisolid contents of the tumor. Care must be
} 
exercised in applying suction only when the catheter is firmly embedded in the tumor to avoid rapid CSF evacuation. Once decompression is accomplished, the bipolar device is again used to dissect the tumor away from the ependymal surface and to coagulate the sites of attachments. Biopsy forceps are then used to remove portions of the tumor bluntly, and by a rotating maneuver the surgeon gently separates the tumor from the walls of the hypothalamus. A combination of these techniques is then used to remove the mass totally and to reestablish CSF circulation through a patent aqueduct. A final inspection of the third ventricle demonstrates the site of resection between the habenular and posterior commissure, with an intact velum interpositum at the roof of the third ventricle.

During endoscopic surgery, continuous irrigation is used to maintain a clear medium for image transmission and for hemostasis. A constant purge is used throughout the procedure to avoid over-insufflating the ventricular system and elevating the intracranial pressure. The simplest method for accomplishing this involves maintaining patency of a portal separate from the working channel. This method thus maintains an intracranial pressure that cannot exceed the pressure of a fluid column equal to the length of the endoscope $\left(15 \mathrm{~cm} \mathrm{H}_{2} \mathrm{O}\right)$. In addition to continuous irrigation, bipolar diathermy and balloon tamponade with a No. 3 French embolectomy catheter are also used for hemostasis. Placement of an external ventricular drain is governed by the degree of intraventricular hemorrhage and an assessment of intraventricular CSF obstruction.

An ETV is performed simultaneously to treat hydrocephalus when a posterior third ventricle tumor or pineal mass is present. If ETV is to be performed, consideration must be given to optimizing each surgical goal. For instance, tumor biopsy sampling preceding ETV may hinder the latter procedure because of poor visualization secondary to hemorrhage. Similarly, using an ideal trajectory into the posterior third ventricle may compromise the best approach for creating an ETV. The ETV is performed before tumor manipulation because of the expectation of some intraventricular hemorrhage and reduced image resolution after attempted tumor resection. This ensures adequate and optimal treatment of hydrocephalus in the event that hemorrhaging from the tumor biopsy procedure creates a visual obstruction. With respect to an entry site, a trajectory is used that approximates a compromise between the two ideal trajectories for each procedure. This entry site typically is located 2 to $3 \mathrm{~cm}$ anterior to the coronal suture. Because these patients may have tumors localized around the third ventricle, the ETV can be more challenging because of the possible deformation of the third ventricle or tumor layering over the third ventricle floor.

\section{RESULTS}

There were 26 patients selected from a larger database who comprised the cohort for this review. These patients ranged in age from 2 months to 21 years (mean 10.5 years), and 22 were male and four were female. Twenty-two patients had hydrocephalus at the time of presentation, and four had normal-sized ventricles. Of these four individuals, one had an incidentally diagnosed colloid cyst, two were being monitored following treatment for a primary malignancy (one with germinoma and one with a primitive neuroectodermal tumor) and a lesion was observed on sur- veillance imaging, and one patient presented with endocrinopathy and a suprasellar mass. Tumor location was classified according to the site of presumed origin; third ventricle in 23 and lateral ventricle in three. Endoscopic procedures performed in these 26 patients included tumor biopsy sampling in 24 and tumor resection in the remaining two individuals. Tumor pathological findings and the respective procedures are summarized in Table 1.

Simultaneous procedures were performed on 14 occasions, including eight ETVs, four endoscopic septostomies, and two tumor cyst decompressions. Therefore, in total 38 procedures were performed in these 26 patients. Stereotaxy was used in seven patients; five of them had tumors situated within the third ventricle that required trajectory planning, and in two there was suspicion of disease recurrence, with the stereotactic investigation being used for localization of the enhancing mass in the lateral ventricle.

The intended endoscopic tumor procedure was successfully accomplished in $25(96 \%)$ of 26 patients. The single failed procedure was an attempted endoscopic biopsy sampling of a pineal region tumor performed in 1998, soon after the endoscopic program was begun. Using a steerable fiberoptic device, no clear visualization of the tumor was obtained because of mild intraventricular hemorrhage. The procedure was thus aborted with no detriment to the patient. Overall, there were no complications from the endoscopic procedures. No patient in this series experienced a postoperative infection, seizures, or clinically recognized hemorrhage.

Externalized ventricular drainage was used in eight patients, seven of whom had significantly raised intracranial pressure at presentation and were thought to be at potential risk for raised intracranial pressure during the postoperative period. These patients underwent either simultaneous ETV (five patients), endoscopic tumor removal with reconstitution of CSF circulation (two), or placement of a ventriculoperitoneal shunt (one) as a definitive method of treatment of hydrocephalus. The externalized drain thus served as a precautionary measure during the postoperative assessment period. In one patient who underwent a colloid cyst resec-

TABLE 1

Pathological findings in 26 pediatric patients who underwent endoscopic management for intraventricular tumors*

\begin{tabular}{lcl}
\hline \hline \multicolumn{1}{c}{ Tumor Type } & No. of Patients & \multicolumn{1}{c}{ Procedure } \\
\hline germ cell tumor & & \\
$\quad$ germinoma & 8 & biopsy \\
$\quad$ yolk sac tumor & 1 & biopsy \\
mature teratoma & 1 & biopsy \\
astrocytoma & 5 & biopsy \\
glioneuronal tumor & 4 & biopsy \\
ATRT & 1 & complete resection \\
craniopharyngioma & 1 & biopsy \\
ependymoma & 1 & biopsy \\
colloid cyst & 1 & biopsy \\
treatment-related changes & 1 & complete resection \\
nondiagnostic biopsy & 1 & biopsy \\
total & 1 & aborted procedure \\
\hline
\end{tabular}

* ATRT $=$ atypical teratoid/rhabdoid tumor; PNET $=$ primitive neuroectodermal tumor. 
tion, an externalized drain was also placed at the time of surgery because of moderate intraventricular hemorrhage.

\section{ILLUSTRATIVE CASES}

\section{Case 1}

This 13-year-old boy presented with 2 months of progressive headaches, vomiting, and a single syncopal episode. His examination was notable for bilateral papilledema and some limitation of upward conjugate gaze. An MR imaging study of the brain revealed dramatic noncommunicating hydrocephalus with a mass in the pineal region that extended into the posterior third ventricle (Fig. 3). A right frontal ETV and tumor biopsy procedure was performed without difficulty by using a precoronal entry site. The histological sample was interpreted as a yolk sac tumor. Biochemical assessment of the CSF revealed an alpha fetoprotein level of $250 \mathrm{ng} / \mathrm{ml}$ and human chorionic gonadotropin of $3 \mathrm{IU} / \mathrm{dl}$, confirming a nongerminomatous germ cell tumor. The patient was subsequently treated with multimodality therapy, including chemotherapy and craniospinal radiation therapy.

\section{Case 2}

This patient was an 18-year-old man who presented with increasingly frequent headaches. Admission MR images indicated noncommunicating hydrocephalus due to a posterior third ventricle mass (Fig. 4A). A right frontal endoscopic approach with stereotactic guidance was used to cannulate the lateral ventricle and to optimize a trajectory below the roof of the third ventricle. The tumor mass was identified and then biopsy samples were obtained before using coagulation. Once an adequate specimen was confirmed, bipolar diathermy and suction aspiration were used for total removal of the friable and relatively avascular tumor. Because clear visualization into the posterior third ventricle confirmed a patent aqueduct, no third ventriculostomy was performed. The pathological interpretation of the tumor was a low-grade glioneuronal lesion. Postoperative imaging indicated a total removal and no further therapy was recommended (Fig. 4B). The patient has had no evidence of disease recurrence or symptoms of hydrocephalus for 22 months.

\section{DISCUSSION}

The use of endoscopy for the management of intraventricular brain tumors has evolved from simply treating the associated hydrocephalus, to sampling of tumor tissue, to tumor resection. The ability to achieve the intended goal is strongly dependent on patient selection, the surgical technique used, and the experience of the surgeon. Thus, when consideration is given to the potential use of endoscopic surgery for an intraventricular brain tumor, one must be very clear about the intended goal and fully realistic regarding the ability to reach that goal.

Findings in the current series highlight one recognizable difference between children and adults. Germ cell tumors and curable benign tumors are all relatively common in children and young adults. The implications of these observations are intuitive. First, due to the frequency of germ cell
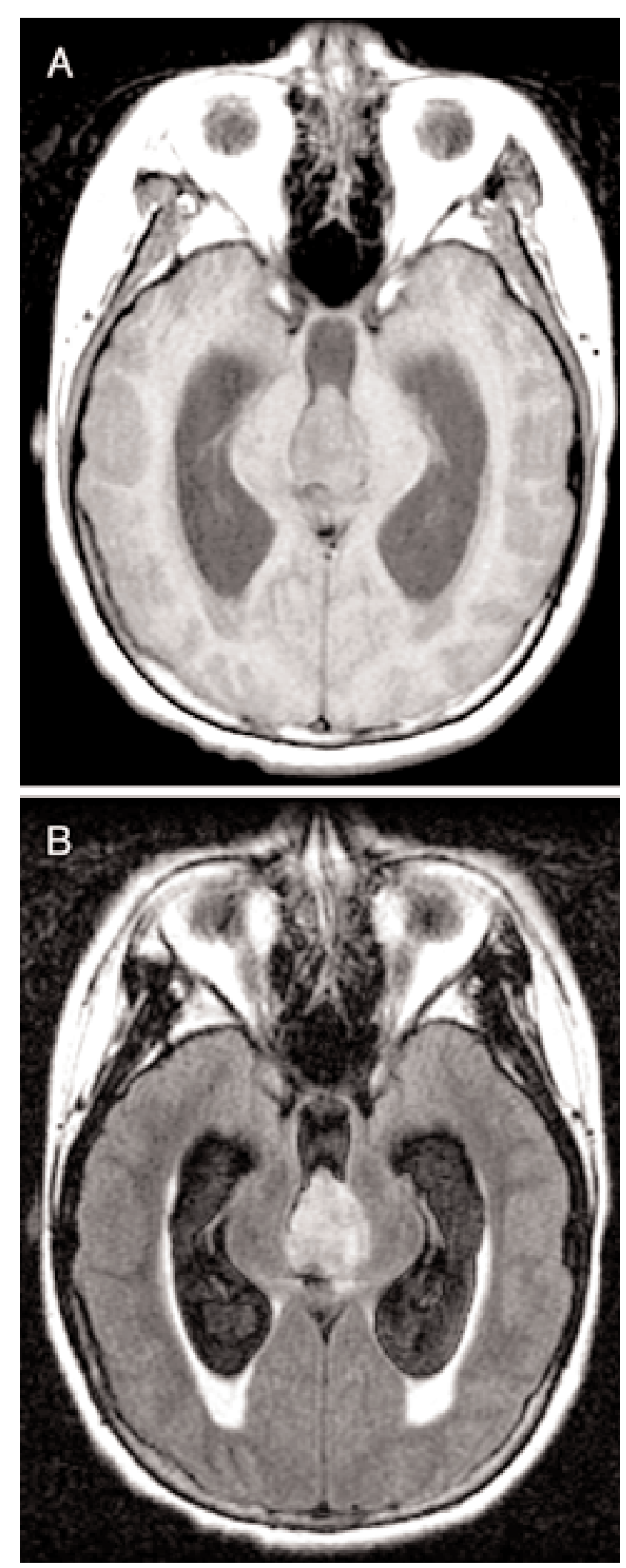

Fig. 3. Axial $\mathrm{T}_{1}$-weighted (A) and fluid-attenuated inversionrecovery (B) MR images obtained in a 13-year-old boy with a pineal region mass obstructing the posterior third ventricle. The severe ventriculomegaly and transependymal signal change are easily appreciated. 

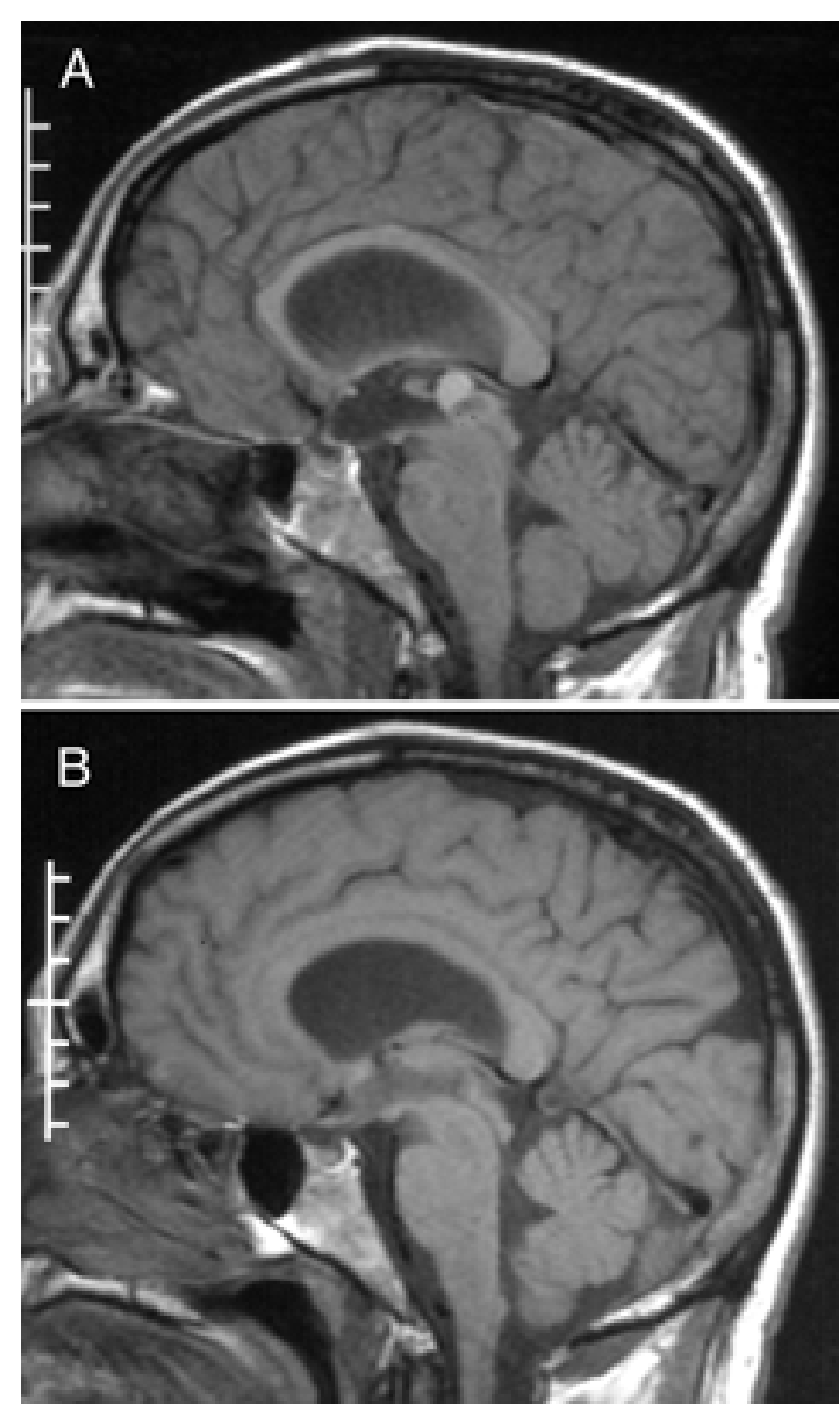

Fig. 4. Sagittal contrast-enhanced, $\mathrm{T}_{1}$-weighted MR images obtained in an 18-year-old man with headaches. The preoperative image (A) depicts a third ventricle mass situated at the posterior third ventricle and obstructing the cerebral aqueduct. The postoperative image (B) confirms a gross-total excision of the mass and complete restoration of the intraventricular CSF circulation.

tumors in children, the use of a minimally invasive technique such as endoscopic biopsy sampling is expected to avoid more invasive biopsy techniques. Furthermore, with the use of biochemical assessment of tumor markers, reliance on craniotomy as an initial procedure for treatment of germ cell tumors should be nearly eliminated. Second, many intraventricular tumors have benign histological features in children; these lesions include ependymoma, lowgrade glial tumors, and choroid plexus papilloma. Because many of these lesions have the potential of being cured with total resection, eligible intraventricular masses can be considered for endoscopic resection, depending on the tumor size, location, and the surgeon's experience.

Endoscopic biopsy is well recognized as an acceptable or preferred technique for tumor sampling in many patients with intraventricular brain lesions. 2,6,7,9,13,15,17,18,22 Many factors, including the surgical technique, the tumor type, and the experience of the pathologist can influence the success of a diagnostic tumor biopsy procedure. In the current series of patients who underwent endoscopic surgery for intraventricular tumors, 24 of the 26 procedures were performed for tissue sampling. In these patients, the overall surgical objective of tumor sampling was successfully achieved in $23(96 \%)$ of 24 . This result represents a significant improvement from most other large studies with a diagnostic yield ranging from 52 to $63 \% .^{6,13,14}$ In their series of 34 patients with pineal region tumors, Pople, et al., ${ }^{17}$ also achieved a better than $90 \%$ diagnostic yield by using an endoscopic technique for tumor biopsy. The single failed biopsy procedure in the current series occurred early in my experience and was due to poor visualization of a posterior third ventricle tumor while using a fiberoptic scope and no navigational guidance. Others have supported the claim that the diagnostic yield is most dependent on the ability to sample tissue rather than the histological interpretation. ${ }^{2,5,18,22}$

Endoscopic tumor removal is most dependent on the composition of the mass. Endoscopic colloid cyst resection has become a very effective and safe method for tumor removal, with many of the advantages inherent in a minimal-

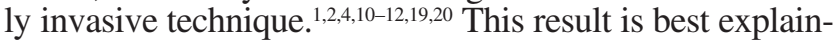
ed by the constituent features of colloid cysts, namely a cystic mass with a thin capsule. Thus, the extirpation is simplified because of the ability to accomplish a significant portion of the removal by aspiration. Solid tumor removal, however, is somewhat limited due to the inadequacy of compatible instrumentation and the small caliber of current endoscopic portals. ${ }^{2,7,13}$ The use of piecemeal removal with cupped biopsy forceps entails prolonged operative times even for relatively small tumors. Instead, the resection of solid tumor is principally achieved through the use of aspiration with a variable suction catheter. Obviously, the success of such a procedure is dependent on the tumor size and composition. The degree of resection relies on the ability to define an interface between the tumor and subjacent white matter. This is defined primarily after visual inspection and is based on the different tissue densities.

In the present series, one patient underwent removal of a solid low-grade tumor in the third ventricle. The success of this removal was no doubt affected by the relatively small size $(<1.5 \mathrm{~cm})$, the friable consistency, and the scarce vascularity of the mass. These three features have been variously commented on in other publications that have addressed the issue of endoscopic tumor removal. In 1998, Gaab and Schroeder ${ }^{7}$ recommended the technique only for lesions with a maximum diameter of less than $2 \mathrm{~cm}$. Although negligible amounts of intraventricular hemorrhage are universal after ventriculoscopy, rarely does the hemorrhage result in patient morbidity or interfere with accomplishing the surgical goal.

Because a majority of patients will present with noncommunicating hydrocephalus, additional endoscopic procedures are frequently required at the time a tumor biopsy or resection is performed. $2,5,13,14,16-18,22$ This requirement further underscores the benefit of using an endoscopic approach in patients with intraventricular tumors. In the current series of 26 patients, 22 had evidence of hydrocephalus at the time of diagnosis. Eight of these underwent successful ETV, four were treated with endoscopic septostomy for a unilateral 
obstruction at the foramen of Monro, two patients underwent cyst decompression with restoration of CSF circulation, and in two there was restoration of normal CSF flow following tumor cyst decompression. The remaining six patients underwent shunt insertion. Thus, $16(73 \%)$ of 22 patients who presented with symptomatic hydrocephalus benefited from endoscopic techniques in avoiding or reducing the shunt burden.

In situations in which bilateral ventricular enlargement exists due to a tumor situated in the anterior third ventricle or at the foramen of Monro, an endoscopic septostomy can eliminate the need for biventricular catheters or for CSF shunting altogether. ${ }^{2}$ Fenestration of the septum pellucidum is easily accomplished with a combination of diathermy, balloon dilation, and sharp dissection. When preparing for endoscopic septostomy, the entry site needs to be modified so that a more lateral trajectory into the ventricle is used. This minor modification reduces the possibility of approaching the septum on a tangential plane, a situation that makes perforation more difficult. A more perpendicular trajectory toward the septum also decreases the risk of injury to structures on the lateral ventricle floor or third ventricle roof.

\section{CONCLUSIONS}

Endoscopic surgery for intraventricular brain tumors has burgeoned over the past decade into a field with clearly defined surgical indications, dedicated instrumentation, and general acceptance by the neurosurgical community. Patient selection requires a thorough understanding of neurooncological principles and the limitations of neuroendoscopic methods. Endoscopic tumor biopsy sampling, colloid cyst resection, and tumor cyst fenestration have been shown to be highly successful while affording significant advantages over conventional neurosurgical techniques. Endoscopic excision of solid intraventricular tumors, although proven to be feasible, remains challenging because of technical limitations. The benefit from using endoscopic techniques to treat intraventricular tumors is further realized by the ability to manage noncommunicating or compartmentalized hydrocephalus by using methods that have clear advantages over shunt placement. Intraoperative guidance contributes greatly toward a safer and more efficacious procedure, and should be considered an integral adjunct in patients undergoing endoscopic neurosurgery for third ventricle brain tumors or in patients without hydrocephalus. The potential usefulness of endoscopic surgery for intraventricular brain tumors is expected to expand with technological advancements in compatible instrumentation. So far, the morbidity associated with endoscopic surgery for intraventricular brain tumors remains minimal, supporting the advantage of this technique over other conventional methods.

\section{References}

1. Abdou MS, Cohen AR: Endoscopic treatment of colloid cysts of the third ventricle. Technical note and review of the literature. J Neurosurg 89:1062-1068, 1998

2. Badie B, Brooks N, Souweidane MM: Endoscopic and minimally invasive microsurgical approaches for treating brain tumor patients. J Neurooncol 69:209-219, 2004
3. Cohen AR: Endoscopic ventricular surgery. Pediatr Neurosurg 19:127-134, 1993

4. Decq P, Le Guerinel C, Brugieres P, et al: Endoscopic management of colloid cysts. Neurosurgery 42:1288-1296, 1998

5. Ferrer E, Santamarta D, Garcia-Fructuoso G, et al: Neuroendoscopic management of pineal region tumors. Acta Neurochir 139:12-21, 1997

6. Fukushima T: Endoscopic biopsy of intraventricular tumors with the use of a ventriculofiberscope. Neurosurgery 2:110-113, 1978

7. Gaab MR, Schroeder HWS: Neuroendoscopic approach to intraventricular lesions. J Neurosurgery 88:496-505, 1998

8. Grunert P, Charalampaki P, Hopf N, et al: The role of third ventriculostomy in the management of obstructive hydrocephalus. Minim Invasive Neurosurg 46:16-21, 2003

9. Grunert P, Hopf N, Perneczky A: Frame-based and frameless endoscopic procedures in the third ventricle. Stereotact Funct Neurosurg 68:80-89, 1997

10. Hellwig D, Bauer BL, Schulte M, et al: Neuroendoscopic treatment for colloid cysts of the third ventricle: the experience of a decade. Neurosurgery 52:525-533, 2003

11. King WA, Ullman JS, Frazee JG, et al: Endoscopic resection of colloid cysts: surgical considerations using the rigid endoscope. Neurosurgery 44:1103-1111, 1999

12. Lewis AI, Crone KR, Taha J, et al: Surgical resection of third ventricle colloid cysts. Preliminary results comparing transcallosal microneurosurgery with endoscopy. J Neurosurg 81:174-178, 1994

13. Macarthur DC, Buxton N, Punt J, et al: The role of neuroendoscopy in the management of brain tumors. Br J Neurosurg 16:465-70, 2002

14. Macarthur DC, Buxton N, Vloeberghs M, et al: The effectiveness of neuroendoscopic interventions in children with brain tumours. Childs Nerv Syst 17:589-594, 2001

15. Oi S, Shibata M, Tominaga J, et al: Efficacy of neuroendoscopic procedures in minimally invasive preferential management of pineal region tumors: a prospective study. J Neurosurg 93: 245-253, 2000

16. Oka K, Yamamoto M, Naggasaka S, et als caused by intraventricular tumors. Childs Nerv Syst 10:162-166, 1994

17. Pople IK, Athanasiou TC, Sandeman DR, et al: The role of endoscopic biopsy and third ventriculostomy in the management of pineal region tumors. Br J Neurosurg 15:305-311, 2001

18. Robinson S, Cohen AR: The role of neuroendoscopy in the treatment of pineal region tumors. Surg Neurol 48:360-367, 1997

19. Rodziewicz GS, Smith MV, Hodge CJ Jr: Endoscopic colloid cyst surgery. Neurosurgery 46:655-662, 2000

20. Schroeder HW, Gaab MR: Endoscopic resection of colloid cysts. Neurosurg 51:1441-1445, 2003

21. Schroeder HW, Gaab MR, Niendrof WR: Neuroendoscopic approach to arachnoid cysts. J Neurosurg 85:293-298, 1996

22. Souweidane MM, Sandberg DI, Bilsky MH, et al: Endoscopic biopsy for tumors of the third ventricle. Pediatr Neurosurg 33: 132-137, 2000

23. Wang JC, Heier L, Souweidane MM: Advances in the endoscopic management of suprasellar arachnoid cysts in children. J Neurosurg 100 (Pediatrics 5):418-426, 2004

Manuscript received April 29, 2005.

Accepted in final form May 31, 2005.

Address reprint requests to: Mark M. Souweidane, M.D., Department of Neurological Surgery, Weill Medical College of Cornell University, 520 East 70th Street, Box 99, New York, New York 10021. email: mmsouwei@med.cornell.edu. 